\title{
Cardiac complications of influenza infection in 3 adults
}

\author{
Tasha Jeyanathan MD, Christopher Overgaard MD, Allison McGeer MSc MD
}

1 57-year-old man was admitted to the coronary intensive care unit with severe pulmonary edema after a 5-day history of influenza-like illness. His medical history included previous tobacco use and a family history of early myocardial infarction. He had received the seasonal influenza vaccine in the fall before presentation.

On examination, he was in marked respiratory distress. His temperature was $38.4{ }^{\circ} \mathrm{C}$, respiratory rate was 33 breaths/min, and oxygen saturation was $86 \%$ on $100 \%$ nonrebreather mask. His blood pressure and heart rate were within normal limits. Respiratory examination revealed diffuse bilateral crackles. An electrocardiogram (ECG) taken on admission showed left bundle branch block, and his cardiac troponin I level was 0.77 (normal <0.07) $\mu \mathrm{g} / \mathrm{L}$. There was no previous ECG available for comparison. Echocardiography showed left ventricular dilatation, moderate hypokinesis with regional variability and an ejection fraction of about $30 \%$ (Figure 1). A radiograph of his chest showed left ventricular dilatation with air-space opacification consistent with moderate pulmonary edema. The differential diagnosis was viral myocarditis with heart failure, acute coronary syndrome with heart failure or community-acquired pneumonia with acute respiratory distress syndrome.

Despite supplemental oxygen and diuresis therapy, the patient's hypoxemia worsened and intubation was required; high-frequency oscillatory support and norepinephrine for blood pressure support were also required. He was given empirical treatment with oseltamivir, ceftriaxone and azithromycin, and dual antiplatelet therapy (acetylsalicylic acid and clopidogrel).

On repeat testing, the patient's cardiac troponin I level was $0.48 \mu \mathrm{g} / \mathrm{L}$, and his creatine kinase level was 2561 (normal < 240) U/L. Influenza A (pH1N1) was detected in bronchoalveolar lavage samples by polymerase chain reaction. All other culture samples were negative. Repeat echocardiography showed no improvement in left ventricular function. His course in hospital was complicated by dyspha- gia, and magnetic resonance imaging showed a small lacunar infarct. He was discharged to a stroke rehabilitation centre 40 days after admission. A persantine cardiolite study performed on an outpatient basis did not show any cardiac ischemia but suggested possible prior myocardial infarction. Diagnostic coronary angiography showed mild to moderate plaque in several small branch vessels but no hemodynamically important atherosclerotic coronary artery disease.

In the second case, a 67-year-old woman presented to the emergency department with a 1 -week history of cough and pleuritic chest pain. Her medical history included hypertension, dyslipidemia, diabetes, obesity and smoking, and she had a family history of coronary artery disease. She had not previously received influenza vaccine. In the emergency department, she had cardiac arrest with pulseless electrical activity that required intubation, mechanical ventilation and 10 minutes of cardiopulmonary resuscitation. An ECG obtained after the arrest showed $0.5 \mathrm{~mm}$ of ST-segment elevation in leads $V_{1}-V_{3}$ and ST-segment depression in the inferolateral lead distribution. Her cardiac troponin I level was elevated $(0.51 \mu \mathrm{g} / \mathrm{L})$. The patient was transferred to our facility for possible emergent percutaneous coronary intervention. Diagnostic coronary angiography showed normal coronary arteries, and left ventricular angiography showed an ejection fraction of about $40 \%$, with anteroapical dyskinesis suggestive of apical ballooning. Her left ventricular

\section{KEY POINTS}

- Cardiac conditions associated with influenza include myocarditis, pericarditis, myocardial infarction, congestive heart failure and sudden death

- Influenza-associated cardiac conditions are typically short-lived and reversible.

- During influenza season, nasopharyngeal swabs for influenza should be obtained from patients with cardiac illness if they have fever or any symptoms compatible with acute respiratory illness.

- Influenza vaccination may be associated with a reduction in the risk of cardiac events, but definitive studies are needed.

\begin{abstract}
Competing interests:
Allison McGeer has performed investigatorplinical trials ded by Sanofi Pasteu and GlaxoSmithKline, as observational studies sponsored by HoffmanLa Roche. She has received payment for lectures from Sanofi Pasteur, Gilead

Sciences, Hoffman-

La Roche and GlaxoSmithKline, and she has received payment for the development of educational presentations from Gilea Sciences. She is on the vanory boards of Sanofi Pasteur, GlaxoSmithKline, (t) Roche and Novartis. No competing interests declared by Tasha Jeyanathan and Christopher
\end{abstract}

This article has been pee reviewed.

Correspondence to

Allison McGeer. amcgeer@mtsinai.on.ca

CMAJ 2013. DOI:10.1503 /cmaj.110807 
end-diastolic pressure was $17 \mathrm{~mm} \mathrm{Hg}$. The patient was thought to have moderate cardiomyopathy secondary to acute catecholamine-mediated injury (Takotsubo cardiomyopathy). The peak troponin I level was $2.36 \mu \mathrm{g} / \mathrm{L}$, and the creatine kinase level was 1193 U/L. The patient's ECG results normalized on day 2, and an echocardiogram obtained on day 5 showed normal left ventricular function, with no evidence of apical ballooning.

A nasopharyngeal swab obtained on admission contained influenza A (pH1N1), as revealed by polymerase chain reaction. There was no other evidence of infection. A metabolic workup, including renal, liver and thyroid function, was within normal limits, and the patient reported no stressful emotional events before presentation. The patient remained hemodynamically stable in hospital, but she required treatment for acute respiratory distress syndrome. She was given oseltamivir for 10 days, transferred back to the referring facility on day 11 , and discharged to a rehabilitation facility on day 32 without further cardiac complications.

In the third case, a previously healthy 25year-old woman, who had not been vaccinated against influenza, presented to hospital with syncope and back pain on day 5 of an influenza-like illness. Physical examination was normal except for sinus tachycardia and dehydration. Chest radiographs and ECG were normal, her troponin $\mathrm{T}$ level was 0.04 (normal < 0.01) $\mu \mathrm{g} / \mathrm{L}$, and her creatine kinase level was $35 \mathrm{U} / \mathrm{L}$. In the emergency department, she was given about $7 \mathrm{~L}$ of intravenous fluid for rehydration. Computed tomographic pulmonary angiography performed 18 hours after admission to rule out pulmonary embolism showed large bilateral pleural effu-

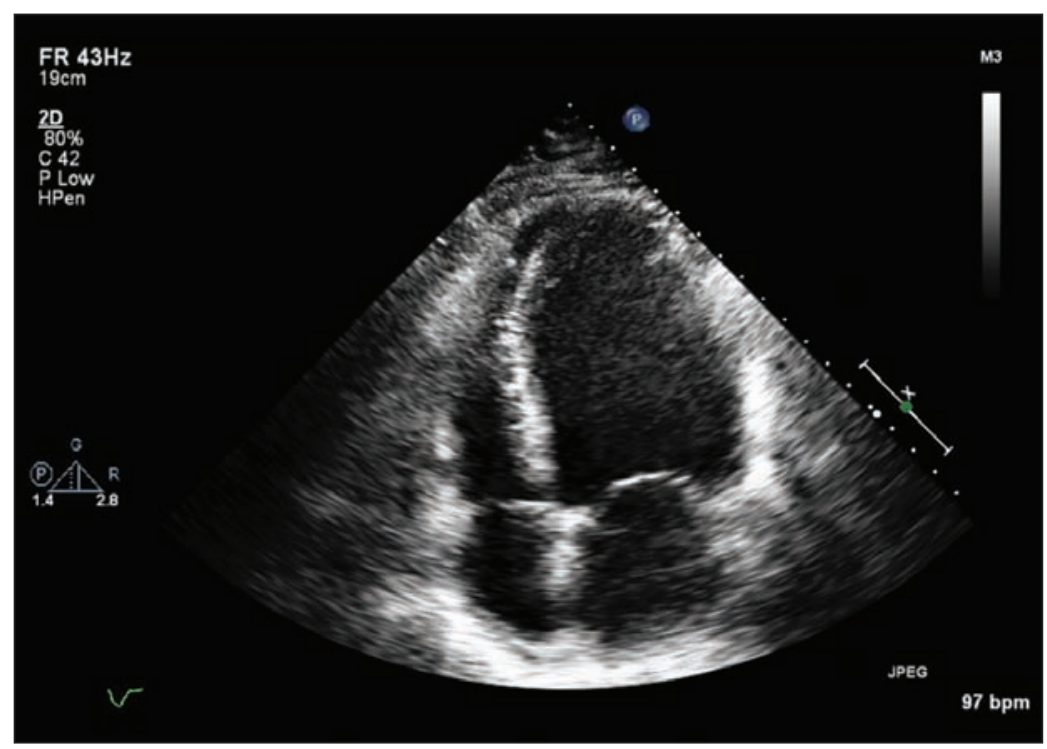

Figure 1: Echocardiogram of a 57-year-old man, with 4-chamber window showing left ventricular dilatation with regional variability. sions and moderate pericardial effusion. Subsequent echocardiography showed a moderate-tolarge circumferential pericardial effusion (maximal width $2 \mathrm{~cm}$ ), without definite evidence of tamponade. A nasopharyngeal swab yielded influenza A (H3N2) by polymerase chain reaction and culture. The patient was given oseltamivir and discharged on day 5. Repeat chest radiography and echocardiography 7 days after discharge showed small residual pleural effusions and a substantial reduction in the size of the pericardial effusion.

\section{Discussion}

The relation between cardiac disease and influenza is complex. Although infection due to human influenza is usually confined to the respiratory tract, myocarditis and pericarditis are well-recognized complications. ${ }^{1}$ The identification of influenza ribonucleic acid in endomyocardial biopsies suggests that myocarditis (including fulminant cardiomyopathy) complicating influenza is caused by disseminated influenza infection. ${ }^{2}$ The finding that influenza RNA has been identified in the myocardium in cases of sudden death, ${ }^{2}$ and a recent study reporting that $15.4 \%$ of deaths due to $\mathrm{pH} 1 \mathrm{~N} 1$ in the United States occurred at home, ${ }^{3}$ suggest that influenza infection may trigger arrhythmias and cardiac arrest. However, the incidence of myocardial involvement in influenza is not known. Older case series with results based on symptoms, ECGs, and measurements of creatine kinase and creatine kinase-muscle and brain (CK-MB) suggested that about $10 \%$ of infected individuals have some cardiac abnormalities. ${ }^{4}$ More recently, Greaves and colleagues ${ }^{5}$ found that creatine kinase was elevated in $8 \%$ of ambulatory young adults with laboratory-confirmed influenza $(n=152)$, but CK-MB was elevated in only $2 \%$, and no patients had elevated levels of cardiac troponins. Similar data are not available for older adults or patients with influenza that required admission to hospital.

Many observational studies have reported consistent associations between influenza and acute myocardial infarction. ${ }^{6}$ A recent analysis by Warren-Gash and colleagues estimated that $3.1 \%-3.4 \%$ of deaths associated with myocardial infarction in England and Wales from 19982008 were attributable to influenza. ${ }^{7}$ In addition, randomized controlled trials provide some evidence that influenza vaccination may reduce the risk of myocardial infarction and cardiovascular death. ${ }^{8}$ The pathophysiology of this effect is not understood. Both clinically defined influenzalike illness and community-acquired pneumonia 
are associated with significant rates of cardiac complications, ${ }^{9}$ and the association with influenza may be specific to infection, or some types of infection, rather than influenza itself.

\section{Clinical course}

As in the cases described in this report, symptoms associated with cardiac complications of influenza are typically (although not invariably) preceded by a 2- to 7-day history of influenzalike or upper respiratory illness. Most cases in Canada occur during a 10- to 18-week period of increased influenza activity ("influenza season") each winter. ${ }^{10}$ Otherwise, the presentations are indistinguishable from cardiac presentations unassociated with influenza. In individual cases, it is not possible to distinguish complications of influenza from cardiac events temporally associated with incidental influenza infection. In myocarditis, common ECG findings include sinus tachycardia, heart block, ST elevation and T-wave inversion; typical echocardiographic findings include globally reduced left ventricular function and pericardial effusion with or without tamponade.

In most cases - as with the second and third patients in our report - cardiac involvement is relatively short-lived and reversible. ${ }^{11,12}$ In our first patient, left ventricular dysfunction persisted for 4 weeks after presentation, and echocardiography showed evidence of regional variability rather than global dysfunction, suggesting that the cardiac abnormality may have resulted from exacerbation of pre-existing coronary disease rather than myocarditis. However, data on the distribution of time to recovery from influenza myocarditis are sparse, and observational data have failed to identify prognostic indicators of time to recovery of left ventricular function after myocarditis. ${ }^{13}$

\section{Prevention}

In this series, 1 of the 3 patients had been vaccinated against influenza. Influenza vaccine prevents about $60 \%$ of uncomplicated symptomatic influenza in healthy younger adults (18-64 yr) and is more effective in preventing severe disease and complications than in preventing uncomplicated disease. ${ }^{14}$ Vaccination is less effective in high-risk and older adults ( $65 \mathrm{yr}$ ). ${ }^{15}$ Although vaccination prevents enough illness and death to be cost-saving to the health care system, many older or chronically ill adults who are vaccinated still require admission to hospital for influenza and its complications. A Cochrane review concluded that there are insufficient data to draw firm conclusions on the effect of vaccination in preventing cardiac complications. ${ }^{8}$

\section{Management}

All of the patients in this report were given antiviral treatment. Whether such treatment is of benefit in preventing or improving the outcome of influenza-associated cardiac dysfunction has not been established. In randomized controlled trials, early treatment of influenza in young healthy adults significantly reduced both viral shedding and the duration and severity of symptoms. ${ }^{16}$ In more severely ill patients with influenza, recent cohort studies have suggested that early antiviral therapy improves clinical outcomes and shortens hospital stay but that late therapy may still provide a treatment benefit. ${ }^{17,18}$ For these reasons, expert guidelines recommend that adults admitted to hospital with suspected or confirmed influenza - either seasonal or pH1N1 - receive antiviral treatment. Moderately ill outpatients, especially those with risk factors for severe illness, and those whose condition is deteriorating may also benefit from antiviral therapy. ${ }^{19,20}$

\section{Conclusion}

There are many presentations of influenzaassociated cardiac dysfunction. During influenza season, complications of influenza may be difficult or impossible to distinguish from primary cardiac disease with incidental influenza. Recognizing the community level of influenza activity and the presence of prodromal symptoms of upper respiratory illness may help clinicians determine which patients require influenza testing. Identifying influenza may be of benefit, both because antiviral therapy may improve patient outcomes and because additional infection-control precautions may prevent transmission of influenza to other patients and staff.

\section{References}

1. Mamas MA, Fraser D, Neyses L. Cardiovascular manifestations associated with influenza virus infection. Int J Cardiol 2008; 130:304-9.

2. Komai T, Nakazawa G, Asai S, et al. Fatal fulminant myocarditis associated with novel influenza A (H1N1) infection. Eur Heart J 2011;32:283

3. Lees CH, Avery C, Asherin R, et al. Pandemic (H1N1) 2009associated deaths detected by unexplained death and medical examiner surveillance. Emerg Infect Dis 2011;17:1479-83.

4. Karjalainen J, Nieminen MS, Heikkila J. Influenza A1 myocarditis in conscripts. Acta Med Scand 1980;207:27-30.

5. Greaves K, Oxford JS, Price CP, et al. The prevalence of myocarditis and skeletal muscle injury during acute viral infection in adults. Arch Intern Med 2003;163:165-8.

6. Warren-Gash C, Smeeth L, Hayward AC. Influenza as a trigger for acute myocardial infarction or death from cardiovascular disease: a systematic review. Lancet Infect Dis 2009;9:601-10.

7. Warren-Gash C, Bhaskaran K, Hayward A, et al. Circulating influenza virus, climatic factors, and acute myocardial infarction: a time series study in England and Wales and Hong Kong. J Infect Dis 2011;203:1710-8.

8. Keller T, Weeda VB, van Dongen CJ, et al. Influenza vaccines for preventing coronary heart disease. Cochrane Database Syst Rev 2008;(3):CD005050.

9. Corrales-Medina VF, Musher DM, Wells GA, et al. Cardiac complications in patients with community-acquired pneumonia: 
incidence, timing, risk factors, and association with short-term mortality. Circulation 2012;125:773-81.

10. Reyes F, Aziz S, Winchester B, et al. Influenza in Canada, 20072008 season update. Can Commun Dis Rep 2008;34:1-9.

11. Erden I, Erden EC, Ozhan H, et al. Echocardiographic manifestations of pandemic 2009 (H1N1) influenza virus infection. J Infect 2010;61:60-5.

12. Martin SS, Hollingsworth CL, Norfolk SG, et al. Reversible cardiac dysfunction associated with pandemic 2009 influenza A (H1N1). Chest 2010;137:1195-7.

13. Ammann P, Naegeli B, Schuiki E, et al. Long-term outcome of acute myocarditis is independent of cardiac enzyme release. Int J Cardiol 2003;89:217-22.

14. Osterholm MT, Kelley NS, Sommer A, et al. Efficacy and effectiveness of influenza vaccines: a systematic review and metaanalysis. Lancet Infect Dis 2012;12:36-44.

15. Couch RB. Prevention and treatment of influenza. $N$ Engl J Med 2000;343:1778-87.

16. Burch J, Corbett M, Stock C, et al. Prescription of anti-influenza drugs for healthy adults: a systematic review and meta-analysis. Lancet Infect Dis 2009;9:537-45.

17. Hsu J, Santesso N, Mustafa R, et al. Antivirals for treatment of influenza: a systematic review and meta-analysis of observational studies. Ann Intern Med 2012;156:512-24.

18. Bramley AM, Dasgupta S, Skarbinski J, et al.; 2009 Pandemic Influenza A (H1N1) Virus Hospitalizations Investigation Team. Intensive care unit patients with 2009 pandemic influenza A
(H1N1pdm09) virus infection - United States. Influenza Other Respi Viruses 2012 June 6. [Epub ahead of print].

19. Harper SA, Bradley JS, Englund JA, et al. Seasonal influenza in adults and children - diagnosis, treatment, chemoprophylaxis, and institutional outbreak management: clinical practice guidelines of the Infectious Diseases Society of America. Clin Infect Dis 2009;48:1003-32.

20. Aoki FY, Allen UD, Stiver HG, et al. The use of antiviral drugs for influenza: guidance for practitioners 2011-2012. Ottawa (ON): Association of Medical Microbiology and Infectious Disease Canada; 2012. Available: www.ammi.ca/media/36413/the $\% 20$ use $\% 20$ of $\% 20$ antiviral $\% 20$ drugs $\% 20$ for $\% 20$ influenza- $\% 20$ website.pdf (accessed 2012 July 17).

Affiliations: From the Department of Medicine (Jeyanathan), University of Toronto; the University Health Network/Mount Sinai Hospital Department of Microbiology (McGeer); and the University Health Network (Overgaard), Peter Munk Cardiac Centre, Toronto, Ont.

Contributors: Tasha Jeyanathan and Christopher Overgaard conceived the idea for this case report. Tasha Jeyanathan performed the literature review and drafted the manuscript. Allison McGeer contributed to the literature review. All authors reviewed and revised the manuscript, and approved the final version submitted for publication. 